\title{
EXISTENCE AND PERTURBATION THEOREMS FOR NONLINEAR MAXIMAL MONOTONE OPERATORS IN BANACH SPACES
}

BY FELIX E. BROWDER

Communicated January 23, 1967

Let $X$ be a Banach space, $X^{*}$ its conjugate space with the pairing between $w$ in $X^{*}$ and $u$ in $X$ denoted by $(w, u)$. If $T$ is a mapping (in general, nonlinear) with domain $D(T)$ in $X$ and range $R(T)$ in $X^{*}$, $T$ is said to be monotone if for all $u$ and $v$ of $D(T)$

$$
(T(u)-T(v), u-v) \geqq 0 .
$$

More generally, a subset $G$ of $X \times X^{*}$ is said to be a monotone set if for each pair $\left[u_{1}, w_{1}\right]$ and $\left[u_{2}, w_{2}\right]$ in $G$, we have

$$
\left(w_{2}-w_{1}, u_{2}-u_{1}\right) \geqq 0 .
$$

Such a set $G$ is said to be maximal monotone if it is maximal among monotone sets in the sense of set inclusion, and a mapping $T$ is said to be maximal monotone if its graph $G(T)$ is a maximal monotone set.

For reflexive Banach spaces $X$ and mappings $T$ with $D(T)=X$, the basic result obtained independently by Browder [2] and Minty [20] states that if $T$ is a monotone operator from $D(T)=X$ to $X^{*}$ which is hemicontinuous, (i.e. continuous from each line segment in $D(T)$ to the weak topology on $X^{*}$ ), and coercive, i.e.

$$
(T u, u) /\|u\| \rightarrow+\infty \quad(\text { as }\|u\| \rightarrow+\infty)
$$

then the range $R(T)$ of $T$ is the whole of the space $X^{*}$. This theorem and its extensions to various classes of operators $T$ from all of $X$ to $X^{*}$ which satisfy modified monotonicity conditions (Browder [4], [9], [10], [11], [12], Leray-Lions [15], Hartman-Stampacchia [14]) are the basis of the application of the theory of monotone operators to obtain general existence theorems for nonlinear elliptic boundary value problems.

For nonelliptic problems, the corresponding reduction to equations for nonlinear operators acting from a reflexive Banach space $X$ to its dual space $X^{*}$ yields operators $T$ which are only defined on a dense subset $D(T)$ of $X$. Results covering the principal parabolic and hyperbolic problems were obtained in Browder [3], [5], [7] for operators $T$ of the form $T=L+T_{0}$, where $T_{0}$ is an everywhere defined hemicontinuous monotone nonlinear operator from $X$ to $X^{*}$ which is coercive and maps bounded subsets of $X$ into bounded sub- 
sets of $X^{*}$ while $L$ is a closed densely-defined monotone linear operator from $X$ to $X^{*}$ such that its adjoint $L^{*}$ is the closure of its restriction to $D(L) \cap D\left(L^{*}\right)$.

It is the purpose of the present note to present some new and much more general results on maximal monotone operators from a reflexive Banach space $X$ to its dual space $X^{*}$ which permit a wider and more flexible application of the theory of densely defined monotone operators. The detailed proofs appear in [13], but we shall develop the principal tool of the argument in Proposition 1 below.

Our basic result is the following.

THEOREM 1. Let $X$ be a reflexive Banach space, $T_{1}$ a maximal monotone mapping from $X$ to $X^{*}$ with $0 \in D\left(T_{1}\right)$. Let $T_{2}$ be a hemicontinuous monotone mapping from all of $X$ to $X^{*}$ which is coercive and maps bounded subsets of $X$ into bounded subsets of $X^{*}$. Let $T=T_{1}+T_{2}$, with $D(T)=D\left(T_{1}\right)$.

Then the range $R(T)$ of $T$ is all of $X^{*}$.

Some important consequences of Theorem 1 are the following:

THEOREM 2. Let $X$ be a strictly convex reflexive Banach space with a strictly convex conjugate space $X^{*}, T_{1}$ a maximal monotone mapping from $X$ to $X^{*}, T_{2}$ a hemicontinuous monotone mapping of all of $X$ into $X^{*}$ which carries bounded subsets of $X$ into bounded subsets of $X^{*}$.

Then the mapping $T=T_{1}+T_{2}$ is a maximal monotone map of $X$ into $X^{*}$.

THEOREM 3. Let $X$ be a reflexive Banach space with a strictly convex dual space and suppose that $T$ is a coercive maximal monotone mapping from $X$ to $X^{*}$ with $0 \in D(T)$.

Then $R(T)$ is all of $X^{*}$.

THEOREM 4. Let $X$ be a uniformly convex Banach space with a strictly convex dual space $X^{*}, T$ a maximal monotone mapping from $X$ to $X^{*}$. Suppose that the inverse image under $T$ of each bounded set is bounded.

Then $R(T)$ is all of $X^{*}$.

The strict convexity and uniform convexity hypotheses of Theorems 2, 3, and 4 can be avoided (as we shall show in another paper) by systematic use of multivalued mappings.

It is shown in [13] that Theorem 1, as well as Theorems 2, 3, and 4, are valid for $T_{1}$ and $T$ multivalued mappings from $X$ to $X^{*}$. This is of particular importance in the application of these results to the subclass of the monotone mappings which consists of the subdifferentials of proper lower semicontinuous convex functions $f$ from $X$ to 
$R^{1} \cup\{+\infty\},([1],[23],[24])$. An element $w$ of $X^{*}$ is said to be a subgradient of $f$ at $u$ in $X$ provided that for all $v$ in $X$

$$
f(v) \geqq f(u)+(w, v-u) .
$$

The subdifferential $\partial f(u)$ is the set of all subgradients $w$ for $f$ at $u$. Rockafellar [24] has shown that for any Banach space $X$ and any proper 1.s.c. convex function $f$ on $X, \partial f$ is a maximal monotone (in general, multivalued) mapping from $X$ to $X^{*}$.

Theorem 5. Let $X$ be a reflexive Banach space with $X^{*}$ strictly convex, $f$ a proper lower semicontinuous convex function on $X$, $\partial f$ its subdifferential. Suppose that $0 \in D(\partial f)$, and let $J$ be a duality mapping of $X$ into $X^{*}$.

Then (a) The mapping $T=\partial f+J$ maps onto $X^{*}$,

(b) If $X$ is uniformly convex, $(\partial f+J)^{-1}$ is a continuous mapping of $X^{*}$ onto $D(\partial f)$. In particular, $D(\partial f)$ is pathwise-connected.

For $X$ a Hilbert space $H$, the result of Theorem 5 is due to Moreau [23], and is related to a theorem of Minty [19] on maximal monotone sets in Hilbert space. For the case treated here, Theorem 5 answers a question posed by Rockafellar in [24].

The proofs of our theorems combine ideas from the study of nonlinear variational inequalities (Browder [11], [12]) and a new approach to the theory of monotone operators on convex sets developed by Minty [22]. (Earlier results on monotone inequalities on convex sets and linear variational inequalities are given in [25], [8], [14], $[10],[16],[17],[18])$. In particular, Minty proves the following (Theorem 3 of $[22]$ ).

Lemma. Let $K$ be a compact convex subset of a locally convex topological vector space $E, G$ a monotone subset of $K \times E^{*}$, wo an arbitrary element of $E^{*}$. Then there exists an element $u_{0}$ of $K$ such that:

$$
\left.\left(w-w_{0}, u-u_{0}\right) \geqq 0, \quad \text { for all }[u, w] \text { in } G\right) \text {. }
$$

We use the following sharper result, which combines Minty's lemma, Lemma 2 of [8], and Lemma 4 of [11]. Its proof, which is based upon that of Lemma 4 of [11], gives an alternative proof of a different type for Minty's lemma.

Proposition 1. Let $K$ be a compact convex subset of a locally convex space $E, G$ a monotone subset of $K \times E^{*}, T$ a continuous map of $K$ into $E^{*}$. Let $w_{0}$ be an element of $E^{*}$.

Then there exists $u_{0}$ in $K$ such that for all $[u, w]$ in $G$,

$$
\left(T\left(u_{0}\right)+w-w_{0}, u-u_{0}\right) \geqq 0 .
$$


Proof of Proposition 1. We may assume without loss of generality that $w_{0}=0$. Suppose that the assertion of Proposition 1 is false. Then for each $x$ in $K$, there exists at least one element $[u, w]$ in $G$ with

$$
(T(x)+w, u-x)<0 .
$$

For each element $[u, w]$ in $G$, let $N_{u, w}$ be the open set in $K$ given by

$$
N_{u, w}=\{x \mid x \in K,(T(x)+w, u-x)<0\} .
$$

The family $\left\{N_{u, w}:[u, w] \in G\right\}$ is then an open covering of the compact set $K$, and hence there exists a finite family $\left\{\left[u_{j}, w_{j}\right], 1 \leqq j \leqq n\right\}$ in $G$ such that the corresponding $N_{u_{j}, w_{j}}$ cover $K$. We form a continuous partition of unity on $K$ corresponding to this finite open covering, i.e. an $n$-tuple $\left\{\beta_{1}, \cdots, \beta_{n}\right\}$ of continuous real functions on $K$ such that for each $j, \beta_{j}$ vanishes outside of $N_{u_{j}, w_{j}}, 0 \leqq \beta_{j}(x) \leqq 1$, and for all $x$ in $K, \sum_{j=1}^{n} \beta_{j}(x)=1$.

We define two continuous mappings $p$ and $q$ of $K$ into $E$ and $E^{*}$, respectively, by:

$$
p(x)=\sum_{j=1}^{n} \beta_{j}(x) u_{j}, \quad q(x)=\sum_{j=1}^{n} \beta_{j}(x) w_{j} .
$$

For each $x$ in $K, p(x)$ is a convex linear combination of points of $K$ and hence lies in $K$. Therefore $p$ is a continuous self-mapping of $K$, and by the Tychonoff fixed point theorem, $p$ has a fixed point $x_{0}$ in $K,\left(p\left(x_{0}\right)=x_{0}\right)$.

On the other hand, if we let

$$
\lambda(x)=(T(x)+q(x), x-p(x)), \quad(x \in K),
$$

and expand $p$ and $q$, we find that $\lambda(x)=\lambda_{1}(x)+\lambda_{2}(x)$, where

$$
\lambda_{1}(x)=\sum_{j=1}^{n} \beta(x)^{2}\left(T(x)+w_{j}, x-u_{j}\right)>0,
$$

for each $x$ in $K$, while:

$$
\lambda_{2}(x)=\sum_{1 \leq j<k_{\S} n} \beta_{j}(x) \beta_{k}(x)\left[\left(T(x)+w_{j}, x-u_{k}\right)+\left(T(x)+w_{k}, x-u_{j}\right)\right] .
$$

We note that:

$$
\begin{aligned}
\left(w_{j}, x-u_{k}\right)+\left(w_{k}, x-u_{j}\right)= & \left(w_{j}, x-u_{j}\right)+\left(w_{k}, x-u_{k}\right) \\
& +\left(w_{j}-w_{k}, u_{j}-u_{k}\right) .
\end{aligned}
$$

Hence for all $x$ in $K$, 


$$
\begin{aligned}
\lambda_{2}(x)=\sum_{j<k} \beta_{j}(x) \beta_{k}(x)[ & \left(T(x)+w_{j}, x-u_{j}\right) \\
& +\left(T(x)+w_{k}, x-u_{k}\right) \\
& \left.+\left(w_{j}-w_{k}, u_{j}-u_{k}\right)\right] \geqq 0 .
\end{aligned}
$$

Finally, $\lambda(x)=\lambda_{1}(x)+\lambda_{2}(x)>0$ for all $x$ in $K$, while

$$
\lambda\left(x_{0}\right)=\left(T\left(x_{0}\right)+q\left(x_{0}\right), x_{0}-p\left(x_{0}\right)\right)=0 .
$$

This contradiction proves the proposition. q.e.d.

The writer has noted that Theorem 5 can also be obtained as a corollary of the results on nonlinear variational inequalities given in Browder [11]. The methods presented here (and developed in detail in [13]) can also be applied to nonlinear variational inequalities involving maximal monotone operators (as well as maximal monotone operators on convex sets) and to more general classes of operators satisfying monotonicity conditions of weaker types.

Added in proof. The writer has discovered that Proposition 1 (and thereby Minty's lemma as well) was proved by H. Debrunner and P. Flor, Archiv. Math. 15 (1964), 445-447, using fixed point theorems for multivalued mappings. The method of proof given above has been applied by the writer to obtain more general fixed point theorems for single-valued and multivalued mappings in topological vector spaces.

\section{REFERENCES}

1. A. Brondsted and R. T. Rockafellar, On the subdifferentiability of convex functions, Proc. Amer. Math. Soc. 16 (1965), 605-611.

2. F. E. Browder, Nonlinear elliptic boundary value problems, Bull. Amer. Math. Soc. 69 (1963), 862-874.

3. - Strongly nonlinear parabolic boundary value problems, Amer. J. Math. 86 (1964), 339-357.

4. - Nonlinear elliptic boundary value prablems. II, Trans. Amer. Math. Soc. 117 (1965), 530-550.

5. - Nonlinear equations of evolution, Ann. of Math. 80 (1964), 485-523.

6. - Multivalued monotone nonlinear mappings and duality mappings in Banach spaces, Trans. Amer. Math. Soc. 118 (1965), 338-351.

7. - Nonlinear initial value problems, Ann. of Math. 82 (1965), 51-87.

8. - Nonlinear monotone operators and convex sets in Banach spaces, Bull. Amer. Math. Soc. 71 (1965), 780-785.

9. - Nonlinear elliptic functional equations in nonreflexive Banach spaces, Bull. Amer. Math. Soc. 72 (1966), 89-95.

10. ——, Problèmes non-linéaires, Univ. of Montreal Press, Montreal, 1966, 153 pp.

11. On the unification of the calculus of variations and the theory of mono- 
tone nonlinear operators in Banach spaces, Proc. Nat. Acad. Sci. U.S.A. 56 (1966), 419-425.

12. - Existence and approximation of solutions of nonlinear variational inequalities, Proc. Nat. Acad. Aci. U.S.A. 56 (1966), 1080-1086

13. - Nonlinear maximal monotone operators in Banach spaces, Math. Ann. (to appear).

14. P. Hartman and G. Stampacchia, On some nonlinear elliptic differential functional equations, Acta Math. 115 (1966), 271-330.

15. J. Leray and J. L. Lions, Quelques résultats de Visik sur les problèmes elliptiques nonlinéaires par les méthodes de Minty-Browder, Bull. Soc. Math. France 93 (1965), 97-107.

16. C. Lescarret, Cas d'addition des applications monotones maximales dans un espace de Hilbert, C. R. Acad. Sci. Paris 261 (1965), 1160-1163.

17. J. L. Lions and G, Stampacchia, Inéquations variationnelles noncoercives, C. R. Acad. Sci. Paris 261 (1965), 25-27.

18. —, Variational inequalities, (to appear).

19. G. J. Minty, Monotone (nonlinear) operatars in Hilbert spaces, Duke Math. J. 29 (1962), 341-346.

20. - On a "monotonicity" method for the solution of nonlinear equations in Banach spaces, Proc. Nat. Acad. Sci. U.S.A. 50 (1963), 1038-1041.

21. - A theorem on maximal monotonic sets in Hilbert space, J. Math. Anal. Appl. 11 (1965), 434-439.

22. - On the generalization of a direct method of the calculus of variations, Bull Amer. Math. Soc. 73 (1967), 315-321.

23. J. J. Moreau, Proximité et dualité dans un espace hilbertien, Bull. Soc. Math. France 93 (1965), 273-299.

24. R. T. Rockafellar, Characterization of the subdifferentials of convex functions, Pacific J. Math. 17 (1966), 497-510.

25. G. Stampacchia, Formes bilinéaires coercitives sur les ensembles convexes, $\mathrm{C} . \mathrm{R}$. Acad. Sci. Paris 258 (1964), 4413-4416.

California Institute of Technology and UNIVERSITY OF CHICAGO 